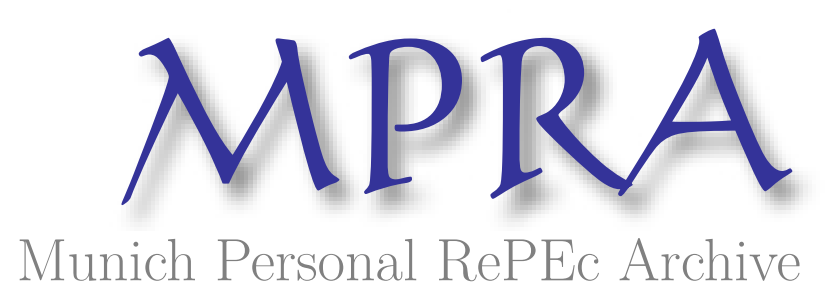

\title{
Certainty equivalent citation: generalized classes of citation indexes
}

Abatemarco, Antonio and Dell'Anno, Roberto

28 July 2011

Online at https://mpra.ub.uni-muenchen.de/38161/

MPRA Paper No. 38161, posted 18 Apr 2012 08:35 UTC 


\title{
Certainty equivalent citation: generalized classes of citation indexes
}

\author{
Antonio Abatemarco ${ }^{a}$, Roberto Dell'Anno ${ }^{b}$ \\ ${ }^{a}$ Department of Economics and Statistics, University of Salerno \\ ${ }^{b}$ Department of Business Economics, Legal, Merceological and Geographical Science, \\ University of Foggia
}

\begin{abstract}
Drawing from the existing literature on risk and inequality measurement, we implement the notion of "certainty equivalent citation" in order (i) to generalize most of the h-type citation indexes (h-, g-, $\tilde{g}$, t-, f-, w-index), and (ii) to highlight the centrality of the decision-maker's preferences on distributive aspects (concentration aversion) for the ranking of citation profiles. In order to highlight the sensitivity of citation orderings with respect to concentration aversion, an application to both simulated and real citation profiles is presented.
\end{abstract}

Keywords: citation index; h-index; w-index; inequality measurement JEL: I23; D63; H52

\section{Introduction}

In the recent years, citation indicators have attracted considerable interest of the scientific community. This is mainly motivated by (i) the increasing need for cost minimization in the allocation of public (and private) research funds (Segalat, 2009), (ii) the recognition of "some role" for citations when evaluating the scientific productivity of a research unit (scholar, department, institute), and (iii) the transparency, comparability of the assessments and criticisms of the peer review system (Weingart, 2005; Bornmann, 2011).

In the existing literature several citation indicators have been proposed (e.g., Moed et al., 1985; Schubert et al., 1987; Hirsch, 2005; Waltman et al., 2011; Albarrán et al., 2011; Leydesdorff et al., 2011; Ravallion and Wagstaff, 2011). In particular, drawing from the recent literature on the pros and cons of the hindex (e.g., Jin, 2006; Bornmann and Daniel, 2007; Rousseau, 2008; Marchant, 2009; Burgos, 2010; Bouyssou and Marchant, 2011; Waltman et al., 2011), several h-type indexes have been defined (e.g., Egghe, 2006a,b; Kosmulski, 2006; 
Woeginger, 2008a; Tol, 2009; Glänzel and Schubert, 2010; Schreiber, 2010). ${ }^{1}$

For our purposes it is worth drawing a separating line between two different groups of h-type indexes. On the one hand, some (h-type) indexes can be fully interpreted as a number of "accredited" publications, that is, the maximum number of publications satisfying minimal citation requirements. Here, citations are used exclusively in order to establish quality requirements and, as a result, the score assigned to each research unit cannot be larger than the number of publications by construction (upper bounded indexes). This is the case of the h-index (Hirsch, 2005), g-index (Egghe, 2006a), $h^{2}$-index (Kosmulski, 2006), w-index (Woeginger, 2008a), and the f- and t-indexes (Tol, 2009). On the other hand, some other (h-type) indexes have been recently proposed by which the qualitative perspective is enforced. Here, the citation indexes do not necessarily identify a number of accredited publications, as the score may be reasonably larger than the number of publications in the presence of few but highly cited scientific outputs. This is the case of the $\tilde{g}$-index (Egghe, 2006b). ${ }^{2}$

In this paper we show that most of the abovementioned citation indexes (h-, $\mathrm{g}-, \tilde{g}, \mathrm{w}-, \mathrm{f}-, \mathrm{t})$ can be generalized by borrowing from the notion of "certainty equivalent income" or "equally distributed equivalent income", which has been largely implemented in the field of risk and inequality measurement (Markowitz, 1952; Atkinson, 1970). Given the definition of "certainty equivalent citation", two generalized classes of citation indexes are presented ( $c^{h}$ - and $c^{w}$-class). As a major result, both classes are characterized by a parameter of concentration aversion capturing the preferences of the decision-maker (e.g., commissioners) with respect to the distribution of citations. The centrality of distributional aspects for the assessment of citation profiles has already been emphasized in Albarrán et al. (2011). ${ }^{3}$ As there may be valid motivations to support either concentration aversion or dispersion aversion, the classes of indexes discussed in this paper may be particularly convenient for the assessment of citation profiles. The paper is organized as follows. The generalized classes of citation indexes are presented in section 2. For each of them, both the upper bounded and unbounded variants are considered. In section 3 an application to both simulated and real data is reported in order to highlight the sensitivity of citation orderings with respect to the concentration aversion parameter. Section 4 provides the conclusion.

\footnotetext{
${ }^{1}$ In this paper we do not consider the indexes complementing the h-index (Jin et al., 2007; Zhang, 2009) as well as the re-scaling procedures suggested in order to overcome some of the major cons of the h-index (Katsaros et al., 2006; Batista et al., 2006; Jin et al., 2007; Schreiber, 2008; Wu, 2010; Ellison, 2010; Harzing, 2011).

${ }^{2}$ As observed in (Woeginger, 2008a), the $\tilde{g}$-index "seems to give the nicest and most natural version of the g-index".

${ }^{3}$ It is worth observing that the same issue had already been highlighted with respect to mean and median citation rate metrics in Tijssen (2002) and Aksnes and Sivertsen (2004). More recently, in order to account for the skewness of citation distributions, Leydesdorff et al. (2011) and Leydesdorff and Bornmann (2011) have submitted new indicators based on a percentile rank approach, while a quadratic influence function has been proposed in Ravallion and Wagstaff (2011) by which a preference for "diminishing marginal influence of citations" is additionally assumed.
} 


\section{Generalized classes of citation indexes}

\subsection{Upper bounded citation indexes}

By "upper bounded" citation indexes we refer to the h-type indexes for which the score cannot be higher than the number of publications by construction. Given the decreasingly ordered citation vector $\left\{x_{1}, \ldots, x_{n}\right\} \in \mathbb{N}_{0^{+}}^{n}$ where $\mathbb{N}_{0^{+}}^{n}$ is the set of non-negative natural numbers such that there exists at least one $i$ with $x_{i}>0,{ }^{4}$ let $c=\left(\frac{1}{n} \sum_{i=1}^{n} x_{i}^{\varepsilon}\right)^{\frac{1}{\varepsilon}}$ be the certainty equivalent citation depending on a parameter $\varepsilon$. In order to make this concept suitable for the construction of h-type metrics, let us consider the following two classes of citation indexes.

Definition 2.1 ( $c^{h}$-class of citation indexes)

Given the decreasingly ordered citation vector $\left\{x_{1}, \ldots, x_{n}\right\} \in \mathbb{N}_{0^{+}}^{n}$ and $k \in[1, n]$,

$$
\begin{aligned}
& c^{h}(x)=\operatorname{Max}\left\{k:\left(\frac{1}{j} \sum_{i=1}^{j} x_{i}^{\varepsilon}\right)^{\frac{1}{\varepsilon}} \geq k \quad \forall j \leq k\right\} \forall \varepsilon \neq 0, \text { and } \\
& c^{h}(x)=\operatorname{Max}\left\{k: \prod_{i=1}^{j} x_{i}^{\frac{1}{j}} \geq k \quad \forall j \leq k\right\} \text { for } \varepsilon=0
\end{aligned}
$$

Definition 2.2 ( $c^{w}$-class of citation indexes)

Given the decreasingly ordered citation vector $\left\{x_{1}, \ldots, x_{n}\right\} \in \mathbb{N}_{0^{+}}^{n}$ and $k \in[1, n]$,

$$
\begin{aligned}
& c^{w}(x)=\operatorname{Max}\left\{k:\left(\frac{1}{j} \sum_{i=1}^{j} x_{i}^{\varepsilon}\right)^{\frac{1}{\varepsilon}} \geq k-j+1 \quad \forall j \leq k\right\} \forall \varepsilon \neq 0, \text { and } \\
& c^{w}(x)=\operatorname{Max}\left\{k: \prod_{i=1}^{j} x_{i}^{\frac{1}{j}} \geq k-j+1 \quad \forall j \leq k\right\} \text { for } \varepsilon=0
\end{aligned}
$$

Using the definition of power mean, or generalized mean, the $c^{h}$-class has been discussed in Schreiber (2010) and Glänzel and Schubert (2010). This class has been shown to mainly generalize the following h-type indexes.

\section{Proposition 2.1}

Given a decreasingly ordered citation vector $\left\{x_{1}, \ldots, x_{n}\right\} \in \mathbb{N}_{0^{+}}^{n}$,

$$
c^{h}(x)=\left\{\begin{array}{lll}
\operatorname{Min}\left\{x_{1}, n\right\} & \text { if } & \varepsilon \rightarrow+\infty \\
g(x) & \text { if } & \varepsilon=1 \\
t(x) & \text { if } & \varepsilon=0 \\
f(x) & \text { if } & \varepsilon=-1 \\
h(x) & \text { if } & \varepsilon \rightarrow-\infty
\end{array}\right.
$$

${ }^{4}$ Equivalently, $x:=\left\{x_{1}, \ldots, x_{n}\right\}$ is an element of the non-negative part of the $n$-dimensional Euclidean space with the origin excluded. 
Proof 2.1 Following Schreiber (2010) and Glänzel and Schubert (2010), (i) if $\varepsilon \rightarrow-\infty$ then $c^{h}(x)=\operatorname{Max}\left\{k: \operatorname{Min}\left\{x_{1}, \ldots, x_{k}\right\} \geq k \forall j \leq k\right\}$ with $1 \leq k \leq n$ that is just equivalent to the $h$-index, and (ii) if $\varepsilon \rightarrow+\infty$, then $c^{h}(x)=\operatorname{Max}\{k$ : $\left.\operatorname{Max}\left\{x_{1}, \ldots, x_{k}\right\} \geq k \forall j \leq k\right\}=\operatorname{Min}\left\{x_{1}, n\right\}$. The rest is straightforward.

As $k \in[1, n]$, the $c^{h}$-class is upper bounded by the number of publications. In addition, compatibly with the definitions of the t-, $\mathrm{f}-$, and h-index, if $\varepsilon \leq 0$ then $c^{h}(x)$ is upper bounded by the number of publications with at least one citation.

Let us now consider Definition (2.2). The latter still implements the notion of certainty equivalent citation, but, in contrast with Definition (2.1), for any possible score of the index $(\mathrm{k})$, the minimal citation requirement (right-hand side) is no longer constant.

\section{Proposition 2.2}

Given a decreasingly ordered citation vector $\left\{x_{1}, \ldots, x_{n}\right\} \in \mathbb{N}_{0^{+}}^{n}$,

$$
c^{w}(x)=\left\{\begin{array}{lll}
\operatorname{Min}\left\{x_{1}, n\right\} & \text { if } & \varepsilon \rightarrow+\infty \\
w(x) & \text { if } & \varepsilon \rightarrow-\infty
\end{array}\right.
$$

Proof 2.2 If $\varepsilon \rightarrow-\infty$ then $c^{w}(x)=\operatorname{Max}\left\{k: \operatorname{Min}\left\{x_{1}, \ldots, x_{k}\right\} \geq k-j+1 \forall j \leq\right.$ $k\}$, that is just the same as the w-index (Woeginger, 2008a). If $\varepsilon \rightarrow+\infty$, then $c^{w}(x)=\operatorname{Max}\left\{k: \operatorname{Max}\left\{x_{1}, \ldots, x_{k}\right\} \geq k-j+1, \forall j \leq k\right\}=\operatorname{Min}\left\{x_{1}, n\right\}=c^{h}(x)$.

Owing to computation similarities, the $c^{w}$-class may be intended as the dual of the $c^{h}$-class. Indeed, when comparing the $\mathrm{w}$ - and h-index, "one computation traverses the publications in increasing order of citations, whereas the other computation does this in decreasing order" (Woeginger, 2008a). In line with the $c^{h}(x)$-class, (i) the $c^{w}(x)$-class is upper bounded by the number of publications, and (ii) if $\varepsilon \leq 0$ then the $c^{w}(x)$-class is upper bounded by the number of publications with at least one citation.

As a major concern, both classes are not independent of the excess citations received by top-cited publications for all $\varepsilon \neq-\infty$, which is known to be one of the major disadvantages of the h-index. In addition, both classes endogenize the value judgments of the decision-maker (e.g., commissioners) with respect to the distribution of citations.

Property 2.1 ( $\varepsilon$-sensitivity to transfers)

Given a citation vector $\left\{y_{1}, \ldots, y_{n}\right\} \in \mathbb{N}_{0^{+}}^{n}$, if $x$ is obtained from $y$ through a non re-ranking citation transfer among publications within the c-core so that, given $y_{j}>y_{i}, x_{i}=y_{i}+\delta$, and $x_{j}=y_{j}-\delta$ with $\delta \geq 1$, then $c^{h}(x) \gtreqless c^{h}(y)$ and $c^{w}(x) \gtreqless c^{w}(y)$ if and only if $\varepsilon \gtreqless 1$.

The decision-maker is concentration averter for all $\varepsilon>1$ but concentration lover for all $\varepsilon<1$. In the case of infinite aversion to concentration $c^{h}(x)=$ $c^{w}(x)=\operatorname{Min}\left\{x_{1}, n\right\}$, whereas in the case of infinite love for concentration, the $c^{h}$ - and $c^{w}$-indexes coincide, respectively, with the h- and w-indexes. Finally, given $\left\{x_{1}, \ldots, x_{n}\right\} \in \mathbb{N}_{0^{+}}^{n}$ with $c^{h}(x) \in[0, n] \forall \varepsilon \in \Re$, the following property holds. 


\section{Property 2.2 (Relative $\varepsilon$-based co-domains)}

Given a decreasingly ordered citation vector $\left\{x_{1}, \ldots, x_{n}\right\} \in \mathbb{N}_{0^{+}}^{n}$, then

$$
\begin{array}{ll}
h(x) \leq f(x) \leq t(x) \leq g(x) \leq c^{h}(x)=\operatorname{Min}\left\{x_{1}, n\right\} & \text { if } \varepsilon \rightarrow+\infty \\
h(x) \leq f(x) \leq t(x) \leq g(x) \leq c^{h}(x) \leq \operatorname{Min}\left\{x_{1}, n\right\} & \text { if } \varepsilon \in] 1,+\infty[ \\
h(x) \leq f(x) \leq t(x) \leq g(x)=c^{h}(x) \leq \operatorname{Min}\left\{x_{1}, n\right\} & \text { if } \varepsilon=1 \\
h(x) \leq f(x) \leq t(x) \leq c^{h}(x) \leq g(x) \leq \operatorname{Min}\left\{x_{1}, n\right\} & \text { if } \varepsilon \in] 0,1[ \\
h(x) \leq f(x) \leq t(x)=c^{h}(x) \leq g(x) \leq \operatorname{Min}\left\{x_{1}, n\right\} & \text { if } \varepsilon=0 \\
h(x) \leq f(x) \leq c^{h}(x) \leq t(x) \leq g(x) \leq \operatorname{Min}\left\{x_{1}, n\right\} & \text { if } \varepsilon \in]-1,0[ \\
h(x) \leq f(x)=c^{h}(x) \leq t(x) \leq g(x) \leq \operatorname{Min}\left\{x_{1}, n\right\} & \text { if } \varepsilon=-1 \\
h(x) \leq c^{h}(x) \leq f(x) \leq t(x) \leq g(x) \leq \operatorname{Min}\left\{x_{1}, n\right\} & \text { if } \varepsilon \in]-\infty,-1[ \\
h(x)=c^{h}(x) \leq f(x) \leq t(x) \leq g(x) \leq \operatorname{Min}\left\{x_{1}, n\right\} & \text { if } \varepsilon \rightarrow-\infty \\
&
\end{array}
$$

with $c^{h}(x) \leq c^{w}(x) \forall \varepsilon .^{5}$

\subsection{Upper unbounded citation indexes}

In this section we briefly discuss the "upper unbounded" variants for the two classes of indexes defined in the previous section.

Definition 2.3 (The $\tilde{c}^{h}$-class of indexes)

Given the decreasingly ordered citation vector $\left\{x_{1}, \ldots, x_{n}\right\} \in \mathbb{N}_{0^{+}}^{n}$ and $k \in \mathbb{N}$,

$$
\begin{aligned}
& \tilde{c}^{h}(x)=\operatorname{Max}\left\{k:\left(\frac{1}{j} \sum_{i=1}^{j} x_{i}^{\varepsilon}\right)^{\frac{1}{\varepsilon}} \geq k \quad \forall j \leq k\right\} \forall \varepsilon \neq 0, \text { and } \\
& \tilde{c}^{h}(x)=\operatorname{Max}\left\{k: \prod_{i=1}^{j} x_{i}^{\frac{1}{j}} \geq k \quad \forall j \leq k\right\} \text { for } \varepsilon=0
\end{aligned}
$$

Definition 2.4 (The $\tilde{c}^{w}$-class of indexes)

Given the decreasingly ordered citation vector $\left\{x_{1}, \ldots, x_{n}\right\} \in \mathbb{N}_{0^{+}}^{n}$ and $k \in \mathbb{N}$,

$$
\begin{aligned}
& \tilde{c}^{w}(x)=\operatorname{Max}\left\{k:\left(\frac{1}{j} \sum_{i=1}^{j} x_{i}^{\varepsilon}\right)^{\frac{1}{\varepsilon}} \geq k-j+1 \quad \forall j \leq k\right\} \forall \varepsilon \neq 0, \text { and } \\
& \tilde{c}^{w}(x)=\operatorname{Max}\left\{k: \prod_{i=1}^{j} x_{i}^{\frac{1}{j}} \geq k-j+1 \quad \forall j \leq k\right\} \text { for } \varepsilon=0
\end{aligned}
$$

\footnotetext{
${ }^{5}$ In addition, it must be the case that $h(x) \leq w(x) \leq 2 h(x)$ (Woeginger, 2008a).
} 
The $\tilde{c}^{h}$ - and $\tilde{c}^{w}$-class of indexes still implement the notion of certainty equivalent citation, but the score assigned to each citation profile is no longer upper bounded by the number of publications. However, compatibly with the $c^{h}$ - and $c^{w}$-class, if $\varepsilon \leq 0$ then $\tilde{c}^{h}(x)$ and $\tilde{c}^{w}(x)$ are upper bounded by the number of publications with at least one citation.

\section{Proposition 2.3}

Given the decreasingly ordered citation vector $\left\{x_{1}, \ldots, x_{n}\right\} \in \mathbb{N}_{0^{+}}^{n}$,

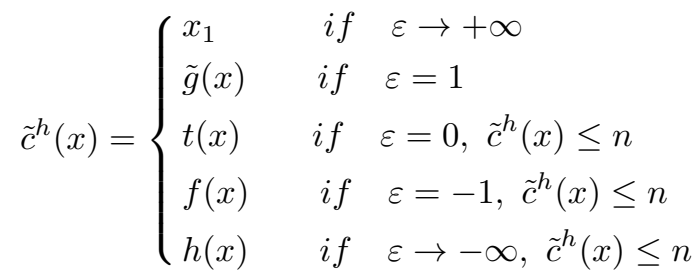

where the $\tilde{g}$-index is the unbounded version of the g-index as proposed by Egghe (2006b).

Proof 2.3 Given $k \in \mathbb{N}$ and

$$
\begin{aligned}
& \lim _{\varepsilon \rightarrow-\infty}\left(\frac{1}{\rho} \sum_{i=1}^{j} x_{i}^{\varepsilon}\right)^{\frac{1}{\varepsilon}}=\operatorname{Min}\left\{x_{1}, \ldots, x_{j}\right\} \forall j \leq n, \forall \rho>1, \\
& \lim _{\varepsilon \rightarrow+\infty}\left(\frac{1}{\rho} \sum_{i=1}^{j} x_{i}^{\varepsilon}\right)^{\frac{1}{\varepsilon}}=\operatorname{Max}\left\{x_{1}, \ldots, x_{j}\right\} \forall j \leq n, \forall \rho>1
\end{aligned}
$$

Proof (1) can be extended to (9) (for $\varepsilon= \pm \infty)$. The rest is straightforward.

\section{Proposition 2.4}

Given the decreasingly ordered citation vector $\left\{x_{1}, \ldots, x_{n}\right\} \in \mathbb{N}_{0^{+}}^{n}$,

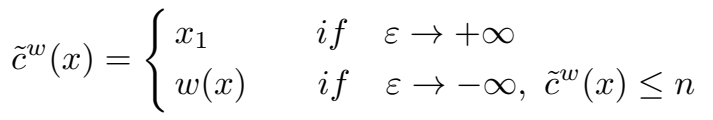

Proof 2.4 Straightforward from Proof (2.2) and Proof (2.3).

Finally, it can be shown that Property (1) ( $\varepsilon$-sensitivity to transfers) still holds in the case of upper unbounded classes of indexes.

\section{Property 2.3 ( $\varepsilon$-sensitivity to transfers)}

Given a citation vector $\left\{y_{1}, \ldots, y_{n}\right\} \in \mathbb{N}_{0^{+}}^{n}$, if $x$ is obtained from $y$ through a non re-ranking citation transfer among publications within the core so that, given $y_{j}>y_{i}, x_{i}=y_{i}+\delta$, and $x_{j}=y_{j}-\delta$ with $\delta \geq 1$, then $\tilde{c}^{h}(x) \gtreqless \tilde{c}^{h}(y)$ and $\tilde{c}^{w}(x) \gtreqless \tilde{c}^{w}(y)$ if and only if $\varepsilon \gtreqless 1$. 


\section{An empirical assessment of the certainty equiv- alent citations}

This section is aimed at demonstrating, by two examples, how the rankings based on the citation indexes (e.g., $c^{h}, c^{w}, \tilde{c}^{h}$, and $\tilde{c}^{w}$ ) depend on the parameter of concentration $(\varepsilon)$ aversion capturing the preferences of the decision-maker. The analysis is carried out by estimating scores and consequent orderings for four hypothetical research units. As re-rankings are more likely to occur only if the units have similar citation profiles, we consider units with the same number of total citations (50) but different distribution of citations over the publications.

$$
\begin{aligned}
& A:=\{5,5,5,5,5,5,5,5,5,5\}, B:=\{12,10,8,7,4,3,2,2,1,1\} \\
& C:=\{25,12,7,3,2,1\}, D:=\{50\}
\end{aligned}
$$

Table 1 depicts the scores of each unit according to different definitions of $\varepsilon$. Rankings are reported in the last column.

[Here: Table 1]

According to this example, an "objective" ranking is out of reach. Depending on the degree of aversion to concentration, re-rankings may occur within the same class of indexes (e.g., see $c^{h}$ - class in Tab.1). From Tab.1 it may be additionally observed that given the same $\varepsilon$, the "upper bounded/unbounded" option is relevant as well (e.g., see $g$ - and $\tilde{g}$-index). As a major concern, it is shown that depending on the decision-maker's preferences, one may move from $A>B>C>D$ to $D>C>B>A$.

As the second example, we consider the real citation records of (two of the three) 2007 Nobel Prize winners in Economics from the Sciverse Scopus in February 2012:

- Prof. Maskin Eric S. who published 55 papers, out of which 48 have been cited at least once, receiving 1446 total citations; and,

- Prof. Myerson Roger who published 48 papers, out of which 43 have been cited at least once, receiving 1484 total citations.

Table 2 reports the scores for each of the four classes of certainty equivalent citation indexes.

[Here: Table 2]

Once again, distributive preferences are found to be clearly relevant for the ranking of the two Nobel Prize winners. 


\section{Concluding remarks}

In the present study, we have drawn a comparison between the notion of "certainty equivalent income" implemented in the field of risk and income inequality measurement (Markowitz, 1952; Atkinson, 1970) and the h-type indexes proposed by Hirsch (2005), Egghe (2006a,b), Woeginger (2008a), and Tol (2009). Within this scenario, we discussed two classes of indexes $\left(c^{h}\right.$ and $c^{w}$ ), which, depending on the value of a parameter of concentration aversion, are shown to replicate well known citation orderings (h-, w-, f-, t-, g-, $\tilde{g}$-index). For each of the two classes, an "upper unbounded" variant is also proposed $\left(\tilde{c}^{h}\right.$ and $\left.\tilde{c}^{w}\right)$ by which the score of the index may reasonably exceed the number of publications in the presence of few but highly cited scientific outputs.

Besides, the sensitivity of both simulated and real citation profiles with respect to distributive aspects has also been shown. In this sense, we wish to contribute to the ongoing debate on the use of citation-based indicators for assessing the impact of research findings by pointing out that, also behind the apparent "impartiality" of citation statistics, there are subjective decision-maker's preferences on the dispersion/concentration of citations (and the upper bounded/unbounded option) that may reasonably affect the rankings of citation profiles. 


\section{References}

Aksnes, D., Sivertsen, G.:, The effect of highly cited papers on national citation indicators, Scientometrics 59, 213-224 (2004).

Albarrán, P., Ortuño, I. and Ruiz-Castillo, J.:, The measurement of low- and high-impact in citation distributions: Technical results, Journal of Infometrics 5, 48-63 (2011).

Atkinson, A.:, On the measurement of inequality, Journal of Economic Theory 2, 244-263 (1970).

Batista, P.D., Campiteli, M.G. and Konouchi, O. :, Is it possible to compare researchers with different scientific interests?, Scientometrics 68 (1), 179-189 (2006).

Bornmann, L., Daniel, H.-D.:, What Do We Know About the h Index?, Journal of the American Society for Information Science and Technology $\mathbf{5 8}$ (9), 1381-1385 (2007).

Bornmann, L.:, Mimicry in science?, Scientometrics 86, 173-177 (2011).

Bouyssou, D., Marchant, T.:, Ranking Scientists and Departments in a Consistent Manner, Journal of the American Society for Information Science and Technology 62, 1761-1769 (2011).

Burgos, A.:, Ranking scientists, Rev. Working Paper Series, 2, Departamento de Fundamentos del Anlisis Econmico, Universidad de Murcia, Spagna (2010).

Egghe, L.:, Theory and practise of the g-index, Scientometrics 69, 131-152 (2006a).

Egghe, L.:, An improvement of the h-index: the g-index, ISSI Newsletter 2, 8-9 (2006b).

Ellison, G.: How Does the Market Use Citation Data? The Hirsch Index in Economics, NBER Working Paper No. 16419, (2010).

Glänzel, W., Schubert, A. :, Hirsch-type characteristics of the tail of distributions. The generalized h-index, Journal of Infometrics 4, 118-123 (2010).

Harzing, A.W.:, Publish or Perish 3.1, www.harzing.com/pop.htm (2011).

Hirsch, J.:, An index to quantify an individual's scientific research output, Proceedings of the National Academy of Sciences, United States of America 102(46), 16569-16572 (2005).

Jin, B.H.: H-index: an evaluation indicator proposed by scientist, Science Focus 1 (1), 8-9 (2006).

Jin, B.H., Liang, L.M., Rousseau, R. and Egghe, L. :, The r- and ar-indices: complementing the h-index, Chinese Science Bulletin 52, 855-863 (2007). 
Katsaros, C., Manolopoulos, Y., Sidiropoulos, A.:, Generalized h-index for disclosing latent facts in citation networks, Scientometrics 72 (2), 253-280 (2006).

Kosmulski, M.:, A new Hirsch-type index saves time and works equally well as the original h-index, ISSI Newsletter 2, 4-6 (2006).

Leydesdorff, L., Bornmann, L.:, Integrated impact indicators compared with impact factors: An alternative research design with policy implications, Journal of the American Society for Information Science and Technology 62, 21332146 (2011).

Leydesdorff, L., Bornmann, L., Mutz, R., Opthof, T.:, Turning the Tables on Citation Analysis One More Time: Principles for Comparing Sets of Documents, Journal of the American Society for Information Science and Technology 62, 1370-1381 (2011).

Marchant, T.:, An axiomatic characterization of the ranking based on the h-index and some other bibliometric rankings of authors, Scientometrics 80, 325-342 (2009).

Markowitz, H.:, Portfolio Selection, Journal of Finance 7, 77-91 (1952).

Moed, H.F., Burger, W.J., Frankfort, J.G. and van Raan, A.F.J.:, The use of bibliometric data for the measurement of university research performance, Research Policy 14, 131-149 (1985).

Ravallion, M. and Wagstaff, A.:, On measuring scholarly influence by citations, Scientometrics 88, 321-337 (2011).

Rousseau, R.:, Reflections on recent developments of the h-index and h-type indices, Collnet Journal of Scientometrics and Information Management $\mathbf{2}$ (2008).

Schreiber, M.:, To share the fame in a fair way, hm modifies h for multi-authored manuscripts, New Journal of Physics 10 (2008).

Schreiber, M.:, A new family of old Hirsch index variants, Journal of Infometrics 4, 647-651 (2010).

Schubert, A., Glänzel, W. and Braun, T.:, Subject field characteristic citation scores and scales for assessing research performance, Scientometrics 12, 267291 (1987).

Segalat, L.:, La science bout de souffle?, Seuil, Paris (2009).

Tijssen, R.M., Visser, M., van Leeuwen, T.:, Benchmarking international scientific excellence: Are highly cited research papers an appropriate frame of reference, Scientometrics 54, 381-397 (2002). 
Tol, R.S.J.:, The h-index and its alternatives: An application to the 100 most prolific economists, Scientometrics 80, 317-324 (2009).

Waltman, L., van Eck, N.J., van Leeuwen, T.N., Visser, M.S., van Raan, A.F.J.:, Towards a new crown indicator: Some theoretical considerations, Journal of Infometrics 5, 37-47 (2011).

Weingart, P.:, Impact of bibliometrics upon the science system: Inadvertent consequences?, Scientometrics 62 (1), 117-131 (2005).

Woeginger, G.J.:, An axiomatic characterization of the Hirsch-index, Mathematical Social Sciences 56, 224-232 (2008a).

Woeginger, G.J.:, An axiomatic analysis of Egghe's g-index, Journal of Infometrics 2, 364-368 (2008b).

Wu, Q.: The w-index: A significant improvement of the h-index, Journal of the American Society for Information Science and Technology 61 (3), 609-614 (2010).

Zhang, C.-T.:, The e-index, complementing the h-index for excess citations, Public Library of Science (PLoS ONE) 4 (5), 1-4 (2009).

Table 1: $c^{h}, c^{w}, \tilde{c}^{h}$ and $\tilde{c}^{w}$ classes of indexes

\begin{tabular}{|c|c|c|cccc|c|}
\hline & & $\varepsilon$ & $\mathrm{A}$ & $\mathrm{B}$ & $\mathrm{C}$ & $\mathrm{D}$ & Rank \\
\hline \multirow{4}{*}{$c^{h}$} & $h-$ & $-\infty$ & 5 & 4 & 3 & 1 & $A>B>C>D$ \\
& $f-$ & -1 & 5 & 5 & 4 & 1 & $A=B>C>D$ \\
& $t-$ & 0 & 5 & 6 & 5 & 1 & $B>A=C>D$ \\
& $g-$ & 1 & 5 & 6 & 6 & 1 & $B=C>A>D$ \\
& $M i n\left(x_{1}, n\right)$ & $+\infty$ & 5 & 10 & 6 & 1 & $B>C>A>D$ \\
\hline \multirow{3}{*}{$c^{w}$} & $w-$ & $-\infty$ & 5 & 8 & 6 & 1 & $B>C>A>D$ \\
& $c^{w}$ & 1 & 5 & 10 & 6 & 1 & $B>C>A>D$ \\
& $M i n\left(x_{1}, n\right)$ & $+\infty$ & 5 & 10 & 6 & 1 & $B>C>A>D$ \\
\hline \multirow{3}{*}{$\tilde{c}^{h}$} & $\tilde{c}^{h}$ & $-\infty$ & 5 & 4 & 3 & 50 & $D>A>B>C$ \\
& $\tilde{g}^{w}$ & 1 & 5 & 6 & 7 & 7 & $D=C>B>A$ \\
& $x_{1}$ & $+\infty$ & 5 & 12 & 25 & 50 & $D>C>B>A$ \\
\hline & $\tilde{c}^{w}$ & $-\infty$ & 5 & 8 & 6 & 50 & $D>B>C>A$ \\
& $\tilde{c}^{w}$ & 1 & 5 & 12 & 13 & 13 & $D=C>B>A$ \\
& $x_{1}$ & $+\infty$ & 5 & 12 & 25 & 50 & $D>C>B>A$ \\
\hline
\end{tabular}


Table 2: $c^{h}, c^{w}, \tilde{c}^{h}$ and $\tilde{c}^{w}$ classes of indexes

\begin{tabular}{|c|c|r|cc|c|}
\hline & & $\varepsilon$ & A: Maskin & B: Myerson & Rank \\
\hline \multirow{4}{*}{$c^{h}$} & $h-$ & $-\infty$ & 19 & 19 & $A=B$ \\
& $f-$ & -1 & 26 & 24 & $A>B$ \\
& $t-$ & 0 & 30 & 27 & $A>B$ \\
& $g-$ & 1 & 37 & 38 & $A<B$ \\
& $\operatorname{Min}\left(x_{1}, n\right)$ & $+\infty$ & 48 & 43 & $A>B$ \\
\hline \multirow{3}{*}{$c^{w}$} & $w-$ & $-\infty$ & 37 & 32 & $A>B$ \\
& $c^{w}$ & 1 & 48 & 43 & $A>B$ \\
& $M i n\left(x_{1}, n\right)$ & $+\infty$ & 48 & 43 & $A>B$ \\
\hline \multirow{3}{*}{$\tilde{c}^{h}$} & $\tilde{c}^{h}$ & $-\infty$ & 19 & 19 & $A=B$ \\
& $\tilde{g}^{-}$ & 1 & 37 & 38 & $A<B$ \\
& $x_{1}$ & $+\infty$ & 199 & 466 & $A<B$ \\
\hline \multirow{2}{*}{$\tilde{c}^{w}$} & $\tilde{c}^{w}$ & $-\infty$ & 37 & 32 & $A>B$ \\
& $\tilde{c}^{w}$ & 1 & 74 & 75 & $A<B$ \\
& $x_{1}$ & $+\infty$ & 199 & 466 & $A<B$ \\
\hline
\end{tabular}

